\title{
CHARM2: An updated Catalog of High Angular Resolution Measurements ${ }^{\star}$
}

\author{
A. Richichi ${ }^{1}$, I. Percheron ${ }^{1}$, and M. Khristoforova ${ }^{1,2}$ \\ ${ }^{1}$ European Southern Observatory, Karl-Schwarzschildstr. 2, 85748 Garching bei München, Germany \\ e-mail: arichich@eso.org \\ 2 Dept. of Astronomy, Physics Faculty, Kazan State University, Kremlevskaya 18, Kazan 420008, Russia
}

Received 21 September 2004 / Accepted 8 October 2004

\begin{abstract}
We present an update of the Catalog of High Angular Resolution Measurements (CHARM, Richichi \& Percheron 2002, A\&A, 386, 492), which includes results available until July 2004. CHARM2 is a compilation of direct measurements by high angular resolution methods, as well as indirect estimates of stellar diameters. Its main goal is to provide a reference list of sources which can be used for calibration and verification observations with long-baseline optical and near-IR interferometers. Single and binary stars are included, as are complex objects from circumstellar shells to extragalactic sources. The present update provides an increase of almost a factor of two over the previous edition. Additionally, it includes several corrections and improvements, as well as a cross-check with the valuable public release observations of the ESO Very Large Telescope Interferometer (VLTI). A total of 8231 entries for 3238 unique sources are now present in CHARM2. This represents an increase of a factor of 3.4 and 2.0, respectively, over the contents of the previous version of CHARM.
\end{abstract}

Key words. techniques: high angular resolution - techniques: interferometric - catalogs - occultations stars: binaries: general - stars: fundamental parameters

\section{Introduction}

The importance of high angular resolution in modern astronomical research can hardly be overestimated. Correspondingly, a renaissance of observational techniques has taken place in the last few decades, taking advantage of technological improvements as well as of new ideas. Recent reviews and details on these issues have been given by Monnier (2003) and Quirrenbach (2001).

The efforts and resources spent on high angular resolution methods and observations has resulted in a wealth of results. While individual measurements have an important role in their specific contexts, it is also important to extract general information from this general knowledge. This includes information with respect to a given class of stars as a whole, or the typical relative accuracy of a type of measurements. Such a global database of high angular resolution measurements was lacking until recently. With CHARM (Richichi \& Percheron 2002, Paper I hereafter), we have attempted to fill this void. CHARM has proven to be a useful tool in particular for the preparation of interferometric observations and in the selection of calibrator sources, as well as of science targets useful for verification purposes.

* The catalog is only available in electronic form at the CDS via anonymous ftp to cdsarc.u-strasbg.fr (130.79.128.5) or via http://cdsweb.u-strasbg.fr/cgi-bin/qcat?J/A+A/431/773
In the three years since the first edition of CHARM, the number of results has grown considerably, as has the fraction of results with stringent observational accuracy. At the same time, the ESO Very Large Telescope Interferometer (VLTI) has completed its cycle of commissioning observations (Schöller et al. 2003) with the VINCI instrument (Kervella et al. 2003), which have been released to the public. The VLTI is among the most powerful interferometers in the world, and ESO is strongly committed to strengthen interferometry as a standard astronomical technique available to the whole community.

We have thus decided to compile an updated edition of CHARM. This also gives us the opportunity to correct and improve a few aspects of the previous edition. As before, CHARM2 is available on line from the Centre de Données Astronomiques de Strasbourg (CDS), as well as on a CD-Rom from the authors with a number of added features.

\section{Upgrades and changes}

In the present edition, CHARM2 includes results available up to July 2004. We have searched the main journals and conference proceedings. Additionally, we have searched on-line databases such as astro-ph (http://xxx.lanl.gov/archive/astro-ph). Dedicated on-line databases have also been used, such as the Optical Long Baseline Interferometry News (OLBIN, http://olbin.jpl.nasa.gov/) and the lunar occultation 
archive maintained by one of us (AR). Our aim has been to be as complete as possible. As a consequence, a very small fraction of the results included may be at a pre-publication stage, and be modified later during the publication process. As before, the original reference is always included (available in full in the CD-Rom version), so that the interested reader can make the final judgement. In this section, we describe the main changes introduced.

\subsection{Changes}

In the first edition, CHARM dealt mostly with three classes of results, namely observations by lunar occultations (LO), longbaseline interferometry (LBI), and estimates by indirect methods such as fits to spectrophotometric data. A few measurements were also present from a variety of other techniques such as speckle interferometry or aperture masking, but for these the coverage was very far from complete. The incidence of these spurious entries was about $2.5 \%$ of the total volume of CHARM. For the new edition, we have decided to add only entries from the first three categories above. We have kept the old entries as they were present in the original CHARM, but we have not included any new results from other noninterferometric techniques. In particular, under interferometry we list only measurements carried out with two or more separate telescopes. Methods that use a single mirror, even with a mask or subapertures, have thus been ignored in the search for new entries.

For the wavelength range, we have now used more restrictions. While the first edition of CHARM included a few measurements recorded in the radio or submillimeter range, new additions in the present edition are restricted to the visual and near-infrared.

We have also decided to remove completely the columns with the estimated angular diameters. This information was computed in the first edition by means of two different empirical calibrations, based on the colors and magnitudes of stellar sources. It was provided mainly as a check, but we have been informed of cases of confusion between the computed diameters and those effectively measured. These latter are of course the main constituent of CHARM2, and we have decided to eliminate the former. Prescriptions for the computation of predicted angular diameters are given for example by Van Belle (1999).

Photometric entries have been revised and expanded, as explained in Sect. 2.4.

\subsection{VLTI measurements}

In March 2001, the ESO VLTI was inaugurated. Since then, an intense program of commissioning observations has been carried out almost uninterruptedly (Schöller et al. 2003). Almost all of the observations carried out on the sky have been made public, and are available on the ESO VLTI web site.

CHARM has been created mainly to facilitate the selection of calibrator stars for large interferometers and to enumerate the high angular resolution observations already available for inteferometric science targets. There is thus a significant overlap between CHARM entries and the set of VLTI commissioning sources. In the present version we explicitly mark all CHARM2 entries which have been observed by the VLTI.

To this purpose, we have used all publicly available commissioning, science demonstration and calibrator data, either with the VINCI or with the MIDI (Leinert et al. 2003) instruments. A total of 275 sources in CHARM2 have corresponding VLTI observations, covering 1458 entries, or about $18 \%$ of the whole catalog. Note that in CHARM2 we do not consider observations that are not made public, such as those carried out during guaranteed time or officially approved science runs. Note also that we do not distinguish between MIDI and VINCI, and we do not provide details such as the number of measurements, the baselines or the dates. This information, as well as a more complete list of VLTI public observations, is provided on the VLTI web pages at ESO.

\subsection{Corrections}

A small number of errors that were present in the first edition have now been corrected. Several LO entries in the first edition of CHARM were drawn from the private archive of one of us (AR). Several of these entries have now been modified, mostly as the result of a refined analysis and/or of final publication.

Some of the entries in CHARM were extracted from papers which were not yet published. These entries have been revised with the final reference. A few errors were also present in the links available in the CD-Rom version, and have now been corrected.

\subsection{Photometry}

The first version of CHARM reported $V$ and $K$ magnitudes for $92.6 \%$ and $93.3 \%$ of the sources, respectively. The $K$ magnitudes however were not always very reliable. Since the publication of the first version of CHARM, two important infrared photometric surveys have been completed and made available, namely 2MASS and DENIS (Cutri et al. 2003, Denis Consortium 2003). We also note that Kimeswenger et al. (2004, KLR04 hereafter) published a survey of DENIS measurements of bright sources, dedicated to selected stars of interest to the VLTI. Also, Bordé et al. (2002) provided $K$ magnitudes for the stars in their catalog of interferometric calibrators.

We have now completely revised the $K$ magnitudes in CHARM2 with the data from these surveys. In particular, we have used an automated search for 2MASS $K$ magnitudes. Since these are not very accurate for saturated sources, we have substitued DENIS $K$ magnitudes from KLR04, when available. Bordé et al. (2002) magnitudes have been used if either 2MASS or KLR04 data were not available. Finally, the $K$ magnitudes from the old CHARM have been kept for those sources not covered by any of the above.

It should be noted that the agreement between all these photometric sources is generally quite acceptable: for example, the average difference between photometry common between 2MASS and KLR04 for 1254 sources is only 0.07 mag. 
However in some cases significant differences exist, that can reach well over one magnitude for some sources. These can be generally explained by intrinsic variability of the source, although systematic differences may not be excluded, especially for bright sources.

Finally, we have introduced a column of $12 \mu \mathrm{m}$ fluxes in CHARM2. These has been found useful expecially from the point of view of mid-infrared interferometric instruments such as MIDI (Leinert et al. 2003). The $12 \mu \mathrm{m}$ fluxes, expressed in Jy, have been extracted from the IRAS-PSC or the MSX Catalogs, in this order.

The level of completeness of photometric entries in CHARM2 is $97.1 \%, 99.7 \%$ and $66.0 \%$ for the $V, K$ and $12 \mu \mathrm{m}$ bands, respectively.

\subsection{Catalog format}

As in the first version, CHARM2 is available from Centre de Données Astronomiques de Strasbourg (CDS), as well as a stand-alone package based on an Excel spreadsheet with hyperlinks to literature entries, statistics, and other information. Also a hyperlinked user manual is provided. This scheme provides the user with the possibility to sort and filter the entries, and to perform calculations such as predicted visibility, circumstances of observability, and other useful features. The full package is available from the authors as a CD-Rom.

The main differences to the previous edition are the addition of a column for the VLTI flags, a column of $12 \mu$ m fluxes and the removal of the columns with the computed diameters. Otherwise, the format of the catalog available on-line from CDS is the same as that described in Paper I.

\section{Results and statistics}

A total of 268 references have been used to compile CHARM2, of which 24 come from the VLTI. The total number of entries is 8231. In many cases more than one entry might be present for a given source, either because measurements were obtained at various wavelengths, or because results were presented by different authors. Note that we always tried to use the same name for an entry as used in the corresponding publication, therefore a source can be present in CHARM 2 under different names. For this, it is helpful to use the list of cross-identifications and associations provided separately. A total of 3238 unique sources are present in CHARM2. Among the sources with the most multiple entries are $\alpha$ Tau, $\alpha$ Aql, $o$ Cet (i.e. Aldebaran, Altair and Mira), with 67, 65 and 60 entries respectively. Entries are classified according to a code for the type of measurement, as listed in Table 1 . These codes are specific for a particular entry as from the original publication, and the same source might fall under different codes accordingly.

In Fig. 1 we show a histogram of the number of publications in two-year bins. It can be easily appreciated how the results in the field of high angular resolution measurements have been steadily increasing in the last decade. More than 50 publications are available in the last bin, i.e. from 2003 and the first half of 2004, which represents about twice the total number of publications in the year before and up to 1984 .
Table 1. List of source codes used in CHARM2.

\begin{tabular}{llrr}
\hline \hline Code & Type of source & \# Entries & Unique \\
\hline Diam & Diameter measured & 1862 & 627 \\
Bin & Binary or mult. system & 450 & 337 \\
UR & Object unresolved & 956 & 864 \\
Com & Complex Geometry & 213 & 92 \\
Cal & Calibrators & 4750 & 1596 \\
\hline
\end{tabular}

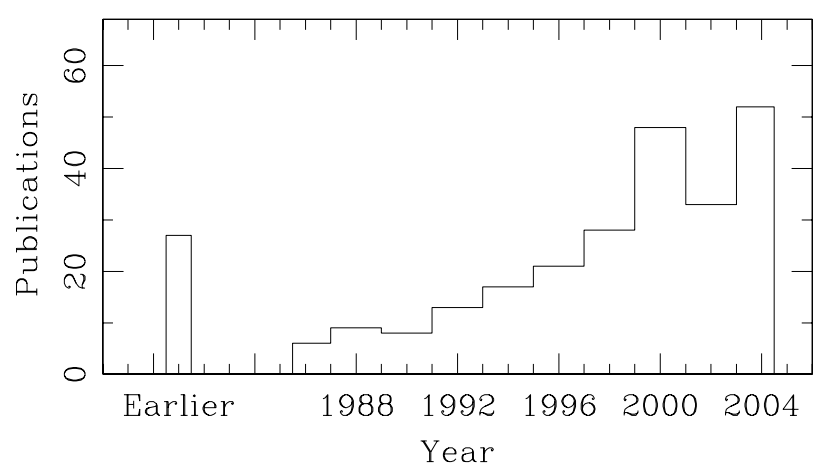

Fig. 1. Distribution of publications included in CHARM2 by year. Each bin is two years wide.

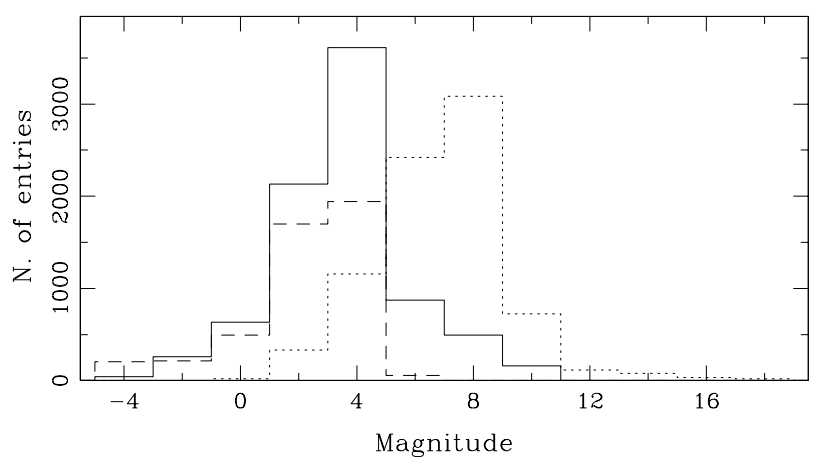

Fig. 2. Distribution of $V, K$ and $12 \mu \mathrm{m}$ magnitudes (dotted, solid and dashed lines respectively).

It is interesting to examine in more detail the general properties of the measurements contained in CHARM2, and we do this through a number of histograms. In Fig. 2 we show the distribution of $V, K$ and $12 \mu \mathrm{m}$ magnitudes (these latter have been converted from the fluxes using a zero point of $28 \mathrm{Jy}$, Hacking et al. 1985).

Both types of infrared magnitudes peak around the value of 4 mag, although the $12 \mu \mathrm{m}$ magnitudes are more skewed with a clear deficit of faint sources, certainly due to a bias in the observational techniques employed. The $V$ magnitudes have an almost Gaussian distribution, with a a peak around $V=8$ mag. The comparison of the $V$ and $K$ distributions clearly illustrates the fact that most of the sources are red in color, a fact due not only to the numerical predominance of such objects in the sky but also to the fact that, for a given total luminosity, a redder stellar disc appears to have a larger angular diameter and is therefore a preferred target for observations. The magnitude distribution is sufficiently wide to provide sources for science verification and calibration of interferometers with quite different characteristics. 


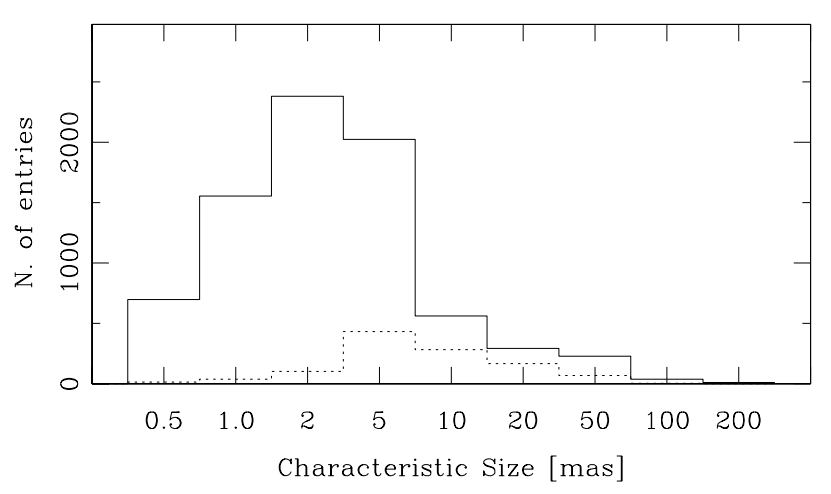

Fig. 3. Distribution of characteristic sizes, as explained in the text. Note that the scale of the horizontal axis is arbitrary. The dotted and solid lines are for CHARM and CHARM2, respectively.

Concerning the distribution of measured angular diameters available in CHARM2, we provide an histogram in Fig. 3. Most of the comments already made in Paper I also apply here. In particular, it should be noted that we have mixed uniform disk and limb-darkened diameters, using the latter when the former were not available. When no diameter information was present, we have used the extension of a circumstellar component, when available. Additionally, no distincion has been made regarding the wavelength of observation. Therefore, the quantities used for the plot should be understood as a characteristic size rather than as a real diameter. It should also be noted that we have made no distinction between those sources which may be represented several times under different entries. Therefore, a bias exists in Fig. 3, but given the large number of measurements present it is nevertheless a sufficient approximation. The peak was around 5 mas for CHARM, but is now closer to 2 mas for CHARM2: this is caused by the large number of measurements contributed by some catalogs for inteferometric calibrators.

We made a similar analysis also for the separation of the binary systems referenced in CHARM2, and we show the result in Fig. 4. Also in this case, many of the comments from Paper I apply. In particular, we have mixed observations of the same source listed under different entries. We also note that in all measurements obtained by LO the values refer to projected separations only. For triple and multiple systems, we have often entered only the separation between the two main components. Although not as large as that for the angular diameters, the statistics of binary systems are relatively large and we can assume that these biases do not affect the general picture too much. In particular one can note a significant difference between Fig. 4 and the similar illustration made in Paper I, i.e., the incidence of binaries with separations below 10 mas has increased in CHARM2 with respect to CHARM, even showing a marked peak around systems with about 5 mas separations. This comes from effective measurements only, and would indicate a higher performance of LO and LBI observations in the last three years.

Binary stars are ideally suited to the verification and calibration of an interferometer, since in this case the visibility does not suffer a monotinic decrease with baseline length as is the case for a resolved stellar disc. Of course, one also should

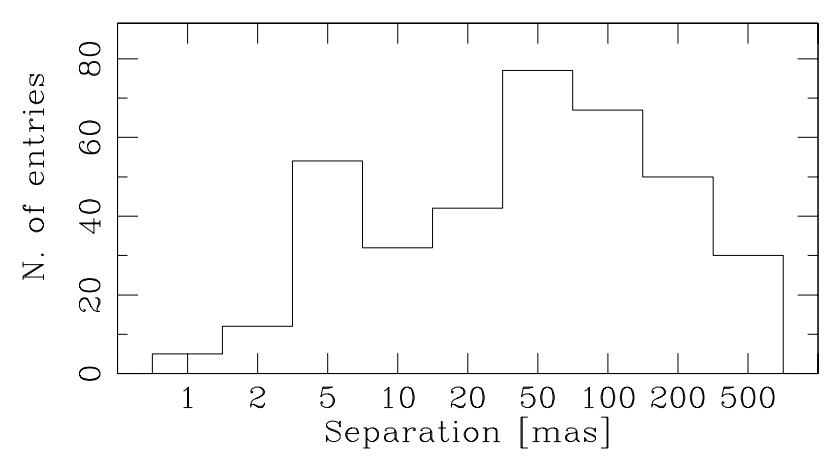

Fig. 4. Distribution of binary separations.

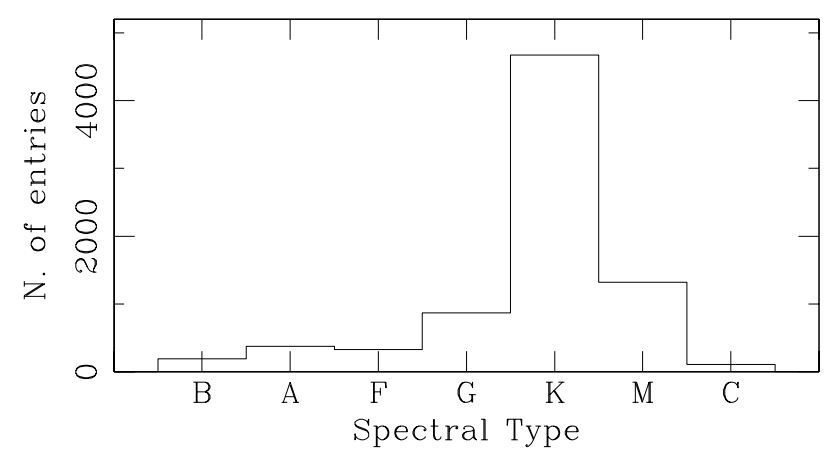

Fig. 5. Distribution of the main spectral types.

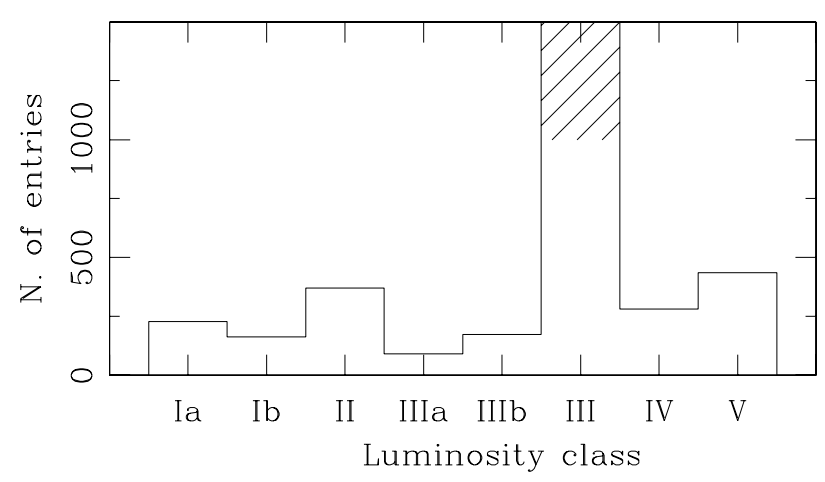

Fig. 6. Distribution of luminosity class. The bin for luminosity class III contains 5181 entries and is shown clipped for clarity.

take into account the brightness ratio of the components in order to assess the feasibility of a measurement.

Finally, in Figs. 5 and 6 we show the distribution of the CHARM2 entries in terms of spectral type and luminosity class. These figures can be compared directly with similar plots provided in Paper I for the previous version of CHARM. The distribution of luminosity class is essentially unchanged. However, the predominance of giant stars included in CHARM2, the distribution of spectral types deserves comment. Although K and M types are by far dominant as before, we notice that the fraction of entries for earlier spectral types has increased significantly. This is due in part to the contribution of catalogs of interferometric calibrators, but also results from facilities with longer baselines than before, as is the case of the VLTI. 


\section{Conclusions}

We have presented an update of the Catalog of High Angular Resolution Measurements, which now includes 8231 entries for 3238 unique sources, compiled from 268 references available to us until July 2004. CHARM2 includes direct measurements by the techniques of lunar occultations and long-baseline interferometry of stellar angular diameters, binary stars and extended sources. It also includes values of stellar angular diameters from indirect methods and catalogs of interferometric calibrators. We have briefly discussed the changes with respect to the previous version of CHARM, and presented statistics for the distributions of magnitudes, characteristic sizes of the objects, binary separations and spectral tpes.

As before, CHARM2 will be available from CDS, with a format similar to the previous version of CHARM. Notable additions are the flags for the presence of publicly available VLTI measurements, and a column of $12 \mu \mathrm{m}$ fluxes. Also the $\mathrm{K}$ magnitudes have been revised and extended. CHARM2 is also available on a CD-Rom from the authors, which includes an Excel version with numerous features including hyperlinks to the full Simbad entries and to the original publications, as well as tools to simulate the observations by an arbitrary interferometer.
Acknowledgements. This research has made use of the Simbad database, operated at the Centre de Données Astronomiques de Strasbourg (CDS), and of NASA's Astrophysics Data System Bibliographic Services (ADS). MK's stay at ESO was financed by the Director General's Discretionary Fund.

\section{References}

Bordé, P., Coudé du Foresto, V., Chagnon, G., Perrin, G. 2002, A\&A, 393, 183

Cutri, R. M., Skrutskie, M. F., Van Dyk, S., et al. 2003, VizieR On-line Data Catalog: II/246

The Denis Consortium 2003, VizieR On-line Data Catalog: B/denis. Cutri, R. M., Skrutskie, M. F., Van Dyk, S., et al. 2003, VizieR On-line Data Catalog: II/246

Hacking, P., Neugebauer, G., Emerson, J., et al. 1985, PASP, 97, 616

Kervella, P., Gitton, P., Segransan, D., et al. 2003, SPIE, 4838, 858K

Kimeswenger, S., Lederle, C., Richichi, A., et al. 2004, A\&A, 413, 1037

Leinert, Ch., Graser, U., Richichi, A., et al. 2003, ESO Messenger, 112,13

Monnier, J. 2003, Rep. Progr. Phys., 66, 789

Quirrenbach, A. 2001, ARA\&A, 39, 353

Richichi, A., Percheron, I. 2002, A\&A, 386, 492, (Paper I)

Schöller, M., Gitton, P., Argomedo, J., et al. 2003, SPIE, 4838, 870S

Van Belle, G. T. 1999, PASP, 111, 1515 